\title{
ON THE USE OF DENSITY-BASED ALGORITHMS FOR THE ANALYSIS OF SOLUTE CLUSTERING IN ATOM PROBE TOMOGRAPHY DATA
}

\author{
Emmanuelle A. Marquis ${ }^{1}$, Vicente Araullo-Peters ${ }^{1}$, Yan Dong ${ }^{1}$, Auriane Etienne ${ }^{2}$, Svetlana \\ Fedotova $^{3}$, Katsuhiko Fujii ${ }^{4}$, Koji Fukuya ${ }^{4}$, Evgenia Kuleshova ${ }^{3}$, Anabelle Lopez ${ }^{5}$, Andrew London 6 , \\ Sergio Lozano-Perez ${ }^{6}$, Yasuyoshi Nagai ${ }^{7}, K_{\text {Kenji Nishida }}^{8}$, Bertrand Radiguet ${ }^{2}$, Daniel Schreiber ${ }^{9}$, \\ Naoki Soneda ${ }^{8}$, Mattias Thuvander ${ }^{10}$, Takeshi Toyama ${ }^{7}$, Faiza Sefta ${ }^{11}$ and Peter Chou ${ }^{12}$ \\ 1. Department of Materials Science and Engineering, University of Michigan, Ann Arbor, MI 48109 U.S.A. \\ 2. Groupe de Physique des Matériaux, UMR CNRS 6634, Univ. de Rouen, Saint Etienne du Rouvray Cedex, France \\ ${ }^{3 .}$ Russian Research Center 'Kurchatov Institute', Pl. Kurtachova, 123182 Moscow, Russian Federation \\ 4. Institute of Nuclear Safety System Inc., 64 Sata, Mihama 919-1205, Japan \\ 5. DEN-Service d'Etudes des Matériaux Irradiés, CEA, Université Paris-Saclay, F-91191, Gif-sur-Yvette, France, \\ France \\ 6. Department of Materials, University of Oxford, Parks Road, Oxford OX1 3PH, UK \\ 7. The Oarai Center, Institute for Materials Research, Tohoku University, Oarai, Ibaraki 311-1313, Japan \\ 8. Materials Science Research Laboratory, Central Research Institute of Electric Power Industry, Nagasaka, Japan \\ 9. Energy and Environment Directorate, Pacific Northwest National Laboratory, Richland, WA 99352, USA \\ 10. Department of Physics, Chalmers University of Technology, SE-412 96, Göteborg, Sweden \\ 11. Departement Métallurgie, EDF-R\&D, Avenue des Renardières-Ecuelles, 77818 Moret-sur-Loing, France \\ 12. Electric Power Research Institute, Palo Alto, CA, 94304, USA
}

\begin{abstract}
Because atom probe tomography (APT) provides three-dimensional reconstructions of small volumes by resolving atomic chemical identities and positions, it is uniquely suited to analyze solute clustering phenomena in materials. A number of approaches have been developed to extract clustering information from the 3D reconstructed dataset, and numerous reports can be found applying these methods to a wide variety of materials questions. However, results from clustering analyses can differ significantly from one report to another, even when performed on similar microstructures, raising questions about the reliability of APT to quantitatively describe solute clustering. In addition, analysis details are often not provided, preventing independent confirmation of the results. With the number of APT research groups growing quickly, the APT community recognizes the need for educating new users about common methods and artefacts, and for developing analysis and data reporting protocols that address issues of reproducibility, errors, and variability issues. To this end, a round robin experiment was organized among ten different international institutions. The goal is to provide a consistent framework for the analysis of irradiated stainless steels using APT. Through the development of more reliable and reproducible data analysis and through communication, this project also aims to advance the understanding between irradiated microstructure and materials performance by providing more complete quantitative microstructural input for modeling. The results, methods, and findings of this round robin will also apply to other clustering phenomena studied using APT, beyond the theme of radiation damage.
\end{abstract}

Keywords: Atom probe tomography, cluster analysis

\section{INTRODUCTION}

Atom probe tomography (APT) has transformed the analysis of irradiated microstructures with its unique chemical and spatial sensitivity that enable the detection of small solute atom clusters. It has provided key insights into the development of solute clusters in irradiated reactor pressure vessel (RPV) steels [1], irradiated austenitic stainless steels [2-4], or oxide dispersion-strengthened steels [5, 6]. APT generates three-dimensional reconstructions of atomic position and chemistry from needle-shaped samples using a combination of field evaporation, time-of-flight mass spectrometry, position-sensitive detection, and a 
reconstruction algorithm. As with any other experimental method, analysis of the APT data requires an understanding of the mechanisms of data generation along with possible limitations and artefacts, a definition of the objects of interest, and a data processing method to quantify the objects. Data generation is beyond the scope of this paper, and we will refer the reader to existing monographs, e.g. [7]. However, it is important to recognize that the physical processes controlling movement and evaporation of atoms under an applied electric field and the limitations inherent to the reconstruction algorithms may result in artefacts and errors in the reconstructed data, with significant consequences on the outcome of the data analysis procedure. Obvious artefacts manifest themselves as variations in the reconstructed atomic density and variations in solute concentrations that depend on crystallographic orientation. Additional sources of errors may be less evident. A number of mechanisms have been identified with significant effects on the spatial and chemical accuracy of the reconstructed data, which includes localized hoping of atoms on the sample surface prior to evaporation (roll-up motion) [9], longer range surface diffusion [8], local changes in the field distribution, particularly near terrace edges [10,11], non-uniform evaporation due to evaporation field differences between phases or crystallographic orientation [12, 13], or detection biased against multiple events [14]. This paper summarizes the round robin experiment on data analysis procedures only; therefore, most of these effects will not be considered.

For density-based methods, solute cluster is defined as a region where solute atoms are spatially closer to one another than they would be when randomly distributed. Two main methods are commonly used to analyze clusters. The first, the "maximum separation method", relies on three parameters $\left(\mathrm{O}, \mathrm{d}_{\max }\right.$, and $\mathrm{N}_{\text {min }}$ ). The order, $\mathrm{O}$, defines the number of nearest neighbors considered. If $\mathrm{O}$ solute atoms are within the critical distance, $\mathrm{d}_{\max }$, of another solute atom, then these atoms are part of the same cluster [15-18]. A cluster requires a minimum number of atoms, $\mathrm{N}_{\min }$, to avoid counting statistically random occurrences of a few atoms being close together. The second, the "local concentration method", relies on an analysis of the local solute concentrations. Clustered atoms are defined by the concentration or density of neighboring solutes being higher than a critical threshold concentration, $C_{\text {th }}$ or number of atoms, $N_{\text {th }}$ [19-21], and by a minimum number of solute atoms, $\mathrm{N}_{\min }$. A combination of both methods, also called the "iso-position method", filters solute atoms with a high local concentration before applying an envelope algorithm [6] or maximum separation method to increase the contrast between clusters and matrix $[4,22]$. Variations on these cluster-finding algorithms, such as Fourier transform and auto-correlation functions [23], pair correlation functions [24, 25], Delaunay tessellation [26], iso-concentration surfaces and proximity histograms [27], and frequency distributions [28] have also been developed for the detection and analysis of solute clusters in APT data; however these are beyond the scope of this round robin experiment.

In either of the two approaches described above, the selection of the analysis parameters is an important step that can significantly modify the outcome of the analysis. A number of studies have been dedicated to defining objective methods of selection. Using a small set of synthetic microstructures, Hyde et al. found that $d_{\max }$ has the largest influence on the outcome [29]. The selection of $\mathrm{N}_{\min }$ and the resulting error on the number density of precipitates were addressed by Cerezo and Davin by comparing the outcomes of the cluster search algorithm performed on an experimental dataset and on a random solid solution. The $\mathrm{N}_{\min }$ value should minimize the number of random occurrences in the matrix $[16,17]$ and Styman et al. refined this step by adding a comparison of cluster compositions in the experimental and randomized datasets [30]. The generation of a random dataset is generally done by re-assigning the measured time-of-flight values at random - as this retains the correct overall composition - while keeping the detected positions, although one should consider the multiplicity of possible random datasets giving rise to a range of possible $\mathrm{N}_{\min }$ values [31]. Different methods have been proposed for the objective selection of a $d_{\max }$ value. These have relied largely on the interpretation of the distribution of nearest neighbor distances (NND) to enable a selection of $\mathrm{d}_{\max }$ that minimizes the inclusion of random clusters, and the splitting or merging of clusters [32]. As a variation on this approach, Jägle proposed to re-examine the NNDs by fitting the part of the distribution pertaining to the matrix rather than selecting a $\mathrm{d}_{\max }$ value based on the comparison with a randomized dataset [33]. Alternatively, Kolli suggested using the variations of the number of clusters to find an optimum value [34]. Following observations that optimized values of $d_{\max }$ and $\mathrm{N}_{\min }$ are correlated [29], Williams et 
al. proposed to compare the observed number of clusters with the expected number of cluster in a randomized dataset by sweeping through a wide range of the parameter space [31].

Despite the number of proposed methods, a challenge remains that no standardized approach to the analysis of solute clusters can be applied, as no single method works for all microstructures of interest. As illustrated below, the maximum separation method is well-suited for microstructures where the solute density contrast between clusters and matrix is significant, but fails when that contrast is reduced. Therefore, not all microstructures will be amenable to this technique and its limitations require clarification. In addition, incomplete reporting of the analysis procedures is apparent in the open literature, raising a range of concerns from data interpretation, validity, to reproducibility. In particular, the absence of details and error or variability analyses limit the reader's assessment of data quality and of appropriateness for comparison against other work or future work.

In this context, the present round robin experiment, conducted among ten international research groups, has several objectives: provide an educational platform for new users, bring awareness about current common methods and their limitations, test the variability of the traditional cluster search methods used by the APT community when applied to irradiated stainless steels, clarify the range of applications suitable to the use of this algorithm, recommend best practices in the use of these methods, and provide a template for reporting data that will assist future researchers in need of re-interpretation of published data.

\section{EXPERIMENTAL}

The current round-robin explores the use of the maximum separation method as implemented in the Cameca software IVAS [35] and the concentration/density methods implemented in the Rouen GPM 3Dsoft software [36]. The maximum separation method or iso-position method can also be performed using open software, such as 3Depict [37]. In order to limit the effects of specimen preparation, data collection, and data reconstruction, a series of synthetic datasets were created to simulate increasingly challenging clustering behaviors. The dataset contained two elements (A and B) where B exhibits clustering tendency and is therefore selected as the core atom in the cluster search algorithms. For each dataset, a fixed number of clusters were randomly distributed in the volume, with a minimum separation distance between clusters. The radii of the clusters were assigned based on a gaussian size distribution. Each cluster was assigned the same concentration with the appropriate number of atoms randomly assigned A or B within the clusters. The only artefact introduced in the data is a random Gaussian translation of the atoms around their atomic lattice sites that is more pronounced for the solute atoms. A translation using a normal distribution with a variance of $\pm \mathrm{a}$ for $\mathrm{x}$ and $\mathrm{y}$ and $\pm \mathrm{a} / 2$ for $\mathrm{z}$ - where $\mathrm{a}$ is the lattice parameter - was then imposed on the positions of all $\mathrm{A}$ atoms and $\mathrm{B}$ atoms in solution. A larger delocalization was applied to B atoms in clusters with a variance of $\pm 2 \mathrm{a}$ for $\mathrm{x}$ and $\mathrm{y}$ and $\pm \mathrm{a}$ for $\mathrm{z}$. To account for the detection efficiency of the currently most used commercial LEAP 4000 HR instrument, $63 \%$ of the atoms were removed at random. Details regarding the datasets can be found in Table 1.

\begin{tabular}{ccccc}
\multicolumn{3}{c}{ Table 1: Cluster characteristics in synthetic datasets (nominal values). } \\
Dataset & $\begin{array}{c}\text { Number of } \\
\text { Clusters }\end{array}$ & $\begin{array}{c}\text { Radius } \\
(\mathrm{nm})\end{array}$ & $\begin{array}{c}\text { Composition of clusters } \\
\text { (matrix) }(\text { at. } \%)\end{array}$ & $\begin{array}{c}\text { Minimum inter- } \\
\text { cluster spacing (nm) }\end{array}$ \\
\hline 1 & 176 & $1.1 \pm 0.1$ & $75(<0.07)$ & 5 \\
\hline 2 & 96 & $1.2 \pm 0.1$ & $20(<0.07)$ & 6 \\
\hline 3 & 275 & $1.2 \pm 0.1$ & $40(<0.07)$ & 6 \\
\hline 4 & 169 & $1.0 \pm 0.1$ & $30(10)$ &
\end{tabular}

The datasets, shown in Fig.1, were distributed to the round robin participants without any prior information regarding their microstructures. Participants were asked to analyze the clustering behavior of B atoms in terms of number of clusters, average cluster size, and average cluster composition. The analysis methods used by the participants were either the maximum separation distance as implemented in the IVAS software or the iso-position method as implemented in the GPM 3Dsoft software. Some participants used iso- 
concentration surfaces (iso-surfaces) to guide their analyses. Atoms on the edges of the volumes were counted as whole or half.
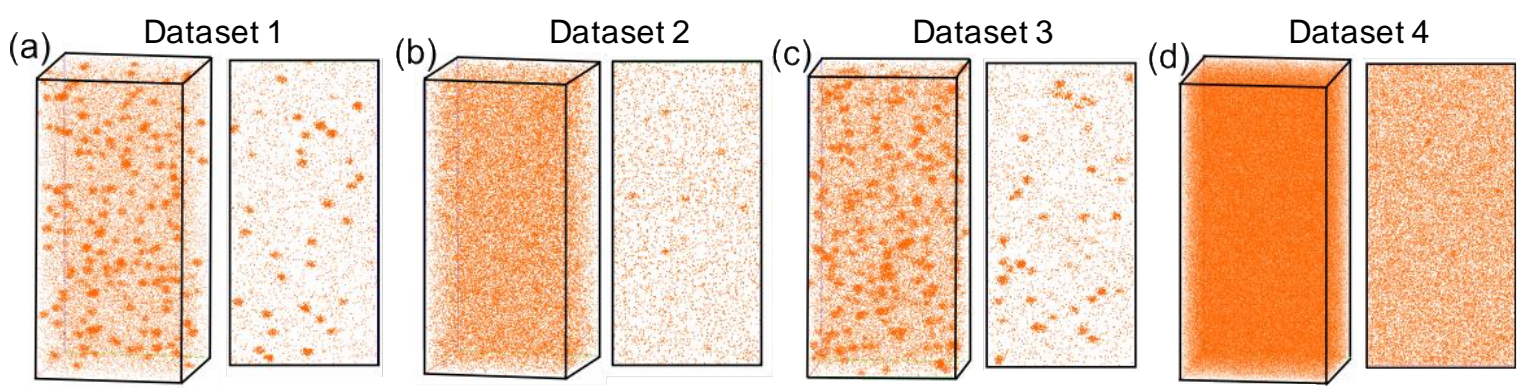

Figure 1: Reconstructions and slices for Dataset 1 (A), Dataset 2 (B), Dataset 3 (C), and Dataset 4 (D). Dataset dimensions are $50 \mathrm{~nm} \times 50 \mathrm{~nm} \times 100 \mathrm{~nm}$. Slices are $10 \mathrm{~nm}$ thick.

\section{RESULTS}

\subsection{Cluster identification}

Dataset 1: The high solute density contrast between clusters and matrix translated into a bimodal distribution of the first nearest neighbor distance (NND); Fig. 2a). For most participants, a comparison with a randomized dataset guided the selection of $d_{\max }$ and $N_{\min }$ values. Some participants selected a higher value of $\mathrm{O}$ without significant effect on the outcome since the contrast between matrix and clusters was already significant with $\mathrm{O}=1$. The values of $\mathrm{N}_{\min }$ ranged from 6 to 20 atoms, without a strong effect on the outcome due to the clear contrast between the size distribution of random clusters and that of the true clusters (Fig. 3). Overall, the stability of this system with respect to the parameters within a relatively wide range of values led to the convergence of the answers; and the number and size of the clusters identified by participants were relatively close to the set value (Table 2). Indeed, a sensitivity analysis with respect to $\mathrm{d}_{\max }$ and $\mathrm{N}_{\min }$ for $\mathrm{O}=1$ showed a wide area of constant cluster density (Fig. 4a). The selection of the $\mathrm{d}_{\max }$ value mostly affected the number of clusters and the measured average cluster size. Fig. 5a illustrates the dependence of the number of detected clusters and size on the $d_{\max }$ value for all choices of $\mathrm{N}_{\min }$ and $\mathrm{O}$ by the participants. Within the stable region, i.e. for $\mathrm{d}_{\max }$ values greater than $0.7 \mathrm{~nm}$, the deviation from the set number of clusters was $\sim 1 \%$, while the size of the cluster was systematically under-estimated by up to $6 \%$. One answer significantly deviated from the others, and was found to be outside the range of stability. Dataset 2: The solute density contrast between matrix and clusters was lower than in the previous case. As illustrated with the NND distributions, a higher order value was required to set the experimental distribution differently enough from the random distribution (Fig. 2b). The sensitivity analysis with respect to $\mathrm{d}_{\max }$ and $\mathrm{N}_{\min }$ at constant $\mathrm{O}$ showed that the number of clusters continuously changes with $\mathrm{d}_{\max }, \mathrm{N}_{\min }$ and did not exhibit a stable region (Fig. 4b). Therefore, a visual check was used by most participants to find the values of the analysis parameters. The reported number of clusters was slightly underestimated. The average of reported cluster size was close to the actual value, with a smaller spread than in Dataset 1 (Table 2). Here, even though the number of clusters was rightly identified using a wide range of $d_{\max }$ values, the narrower range of intermediate $d_{\max }$ values yielded answers close to the nominal value both in terms of number of clusters and cluster sizes (Fig. 5b). Larger value of $\mathrm{d}_{\max }$ tended to include too many matrix ions due to the smaller difference in the NND between the clusters and matrix. The deviation from the set number of clusters was $\sim 10 \%$, while the size of the cluster was overestimated by $16 \%$.

Dataset 3: Here, some clusters were allowed to be closer in space (Fig. 1c), leading to possible merger of clusters through the search algorithm if too large of a $d_{\max }$ value was selected. While an objective selection of $\mathrm{d}_{\max }$ was in principle possible due to the bimodal distributions of NNDs (Fig. 2c), both the number and size of the clusters were highly dependent on the selection of the $d_{\max }$ value (Fig. $\left.4 \mathbf{c}\right)$. The reported numbers of clusters and cluster sizes varied widely, as only a very narrow range of parameter values led to answers close to the nominal value without the use of additional corrections to account for cluster merging. Small 
$\mathrm{d}_{\max }$ values yielded numbers of clusters that were close to the actual number but meanwhile significantly underestimated the cluster size. On the other hand, larger $\mathrm{d}_{\max }$ value merged clusters that were spatially close to each other and therefore overestimated the cluster size. Only one participant manually split clusters when they appeared to be merged.

Dataset 4: The cluster microstructure in this dataset presented the challenge of a high matrix solute concentration. As a result, the distribution of NND failed to show significant deviation from the random distribution, even to orders as high as 10 (Fig. 2d). Participant selection of parameters for $\mathrm{d}_{\max }$ was therefore arbitrary and so were the outcomes, as illustrated with the steep variations with $d_{\max }$ and $N_{\min }$ (Fig. $4 \mathbf{d}$ ). Alternatively, the concentration-based method could be applied to this dataset while maintaining an objective selection of parameter values, and two participants provided answers that were closer to the nominal value. As illustrated in Fig. 6, the use of the local concentration highlighted the clusters while reducing the matrix background. The selection of a maximum separation distance could become objective again, and the comparison with randomized datasets could be used for selecting the threshold concentration (25 at.\% in Fig. 6); the maximum separation method was subsequently applied to the filtered atoms.

\subsection{Cluster composition}

Participants used three different methods to estimate cluster compositions. The inclusion method implemented in the IVAS software requires two additional parameters: an inclusion distance $\mathrm{L} \leq \mathrm{d}_{\max }$, and an erosion distance, $\mathrm{e} \leq \mathrm{L}$, to erode the cluster matrix interface [16]. The value of L should maximize the inclusion of all atoms within the clusters while minimizing neighboring cluster overlap. The second method consisted of calculating radial average concentration profiles from the center of clusters, while the third method relied on generating proximity histograms based on an iso-concentration surface whose position depends on the selection of a bin size and a delocalisation distance [27]. We note that other methods have been described, including the use of NNDs for estimating both matrix and cluster concentrations [38].

For all cases, the reported cluster compositions, summarized in Table 2 and Fig. 7, strongly depended on the method used. The inclusion method performed most poorly with the largest deviations from the set compositions. It appeared to strongly underestimate the higher cluster concentrations while it significantly overestimated the more dilute cluster compositions. The other methods behaved comparably. The compositions reported using the radial concentration method were systematically above the two methods, with a deviation that increased with increasing cluster concentration. Best results were obtained for the intermediate concentrations with deviations up to $15 \%$ from the set values, while deviations of 20 and $25 \%$ were noted for the lower concentration at $20 \%$ and highest concentration at $75 \%$ respectively.

Table 2: Reported cluster search parameter values and cluster characteristics

\begin{tabular}{|c|c|c|c|c|c|}
\hline & 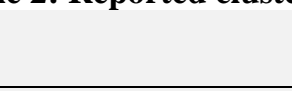 & $\begin{array}{c}\text { Number of } \\
\text { clusters }\end{array}$ & $\begin{array}{l}\text { size (in } \\
\text { atoms) }\end{array}$ & $\begin{array}{c}\text { Cluster } \\
\text { concentration }\end{array}$ & $\begin{array}{c}\text { Matrix } \\
\text { concentration }\end{array}$ \\
\hline \multirow{4}{*}{ Dataset 1} & Nominal & 176 & 139 & 75 & 0.42 \\
\hline & Mean & 178 & 121 & 48.9 & 0.44 \\
\hline & Standard deviation & 5.4 & 16.3 & 11.1 & 0.03 \\
\hline & Spread & 15 & 49.4 & 25.4 & 0.04 \\
\hline \multirow{4}{*}{ Dataset 2} & Nominal & 96 & 30 & 20 & 0.67 \\
\hline & Mean & 86.6 & 35.3 & 24.1 & 0.66 \\
\hline & Standard deviation & 3.8 & 10.3 & 12.7 & 0.01 \\
\hline & spread & 11 & 26 & 33.1 & 0.03 \\
\hline \multirow{4}{*}{ Dataset 3} & Nominal & 275 & 44 & 40 & 0.41 \\
\hline & Mean & 265.5 & 78 & 30.5 & 0.44 \\
\hline & Standard deviation & 23.2 & 22.1 & 10.8 & 0.04 \\
\hline & spread & 83 & 75 & 14.1 & 0.11 \\
\hline \multirow{4}{*}{ Dataset 4} & Nominal & 169 & 30 & 30 & 9.88 \\
\hline & Mean & 103.3 & 89.6 & 44.2 & 9.91 \\
\hline & Standard deviation & 41.8 & 66.2 & 11.3 & 0.06 \\
\hline & Spread & 124 & 186.3 & 31.8 & 0.16 \\
\hline
\end{tabular}



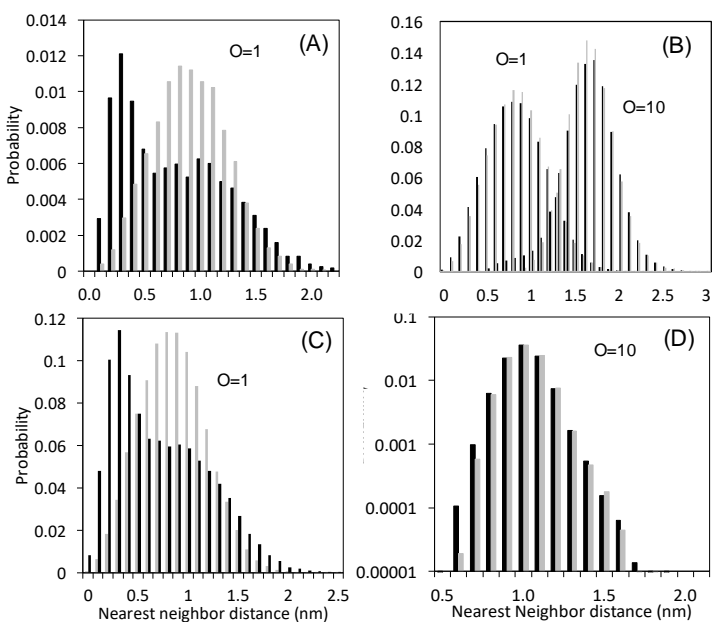

Figure 2: Nearest neighbor distributions for Dataset 1 (A),

Dataset 2 (B), Dataset 3 (C), and Dataset 4 (D). The grey bars represents random distributions.
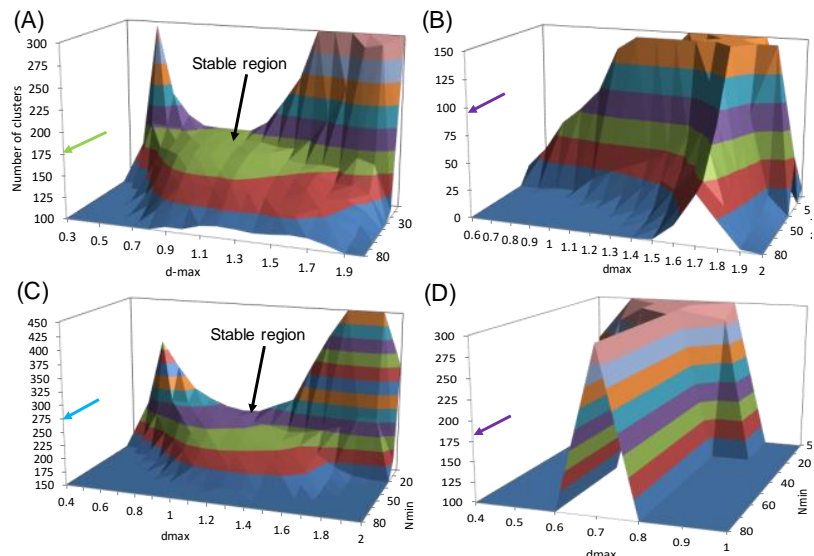

Figure 4: Sensitivity analysis with respect to $d_{\max }$ and $N_{\min }$ for the maximum separation distance, applied to Dataset 1 with $\mathrm{O}=1(\mathrm{~A})$, Dataset 2 with $\mathrm{O}=5(\mathrm{~B})$, Dataset 3 with $\mathrm{O}=$ $1(\mathrm{C})$, and Dataset 4 with $\mathrm{O}=10$ (D). Arrows point to the nominal number of clusters

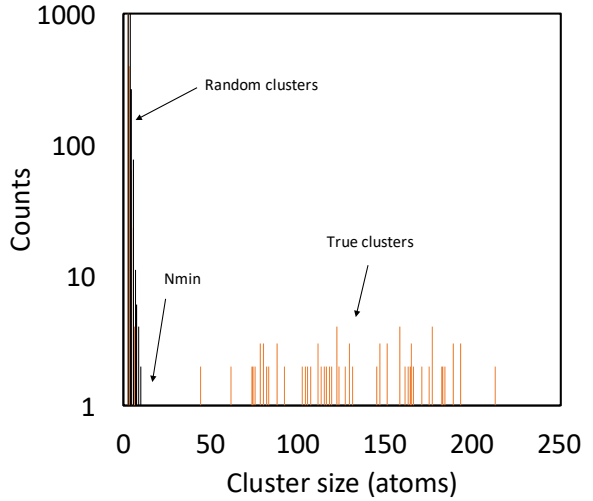

Figure 3: Size distribution of the clusters for dataset $1\left(\mathrm{~d}_{\max }=\right.$ $0.7 \mathrm{~nm}, \mathrm{O}=1$ )
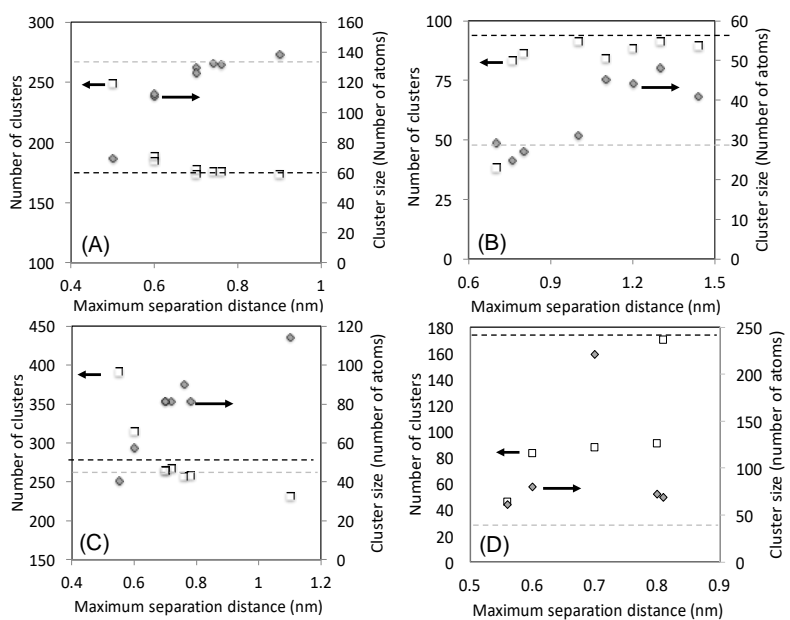

Figure 5: Reported number of clusters and cluster sizes as a function of the selected $d_{\max }$ values in the case of the maximum separation distance for Dataset 1 (A), Dataset 2

(B), Dataset 3 (C), and Dataset 4 (D). Dashed lines corresponds to nominal values, and arrows indicate the relevant axis.
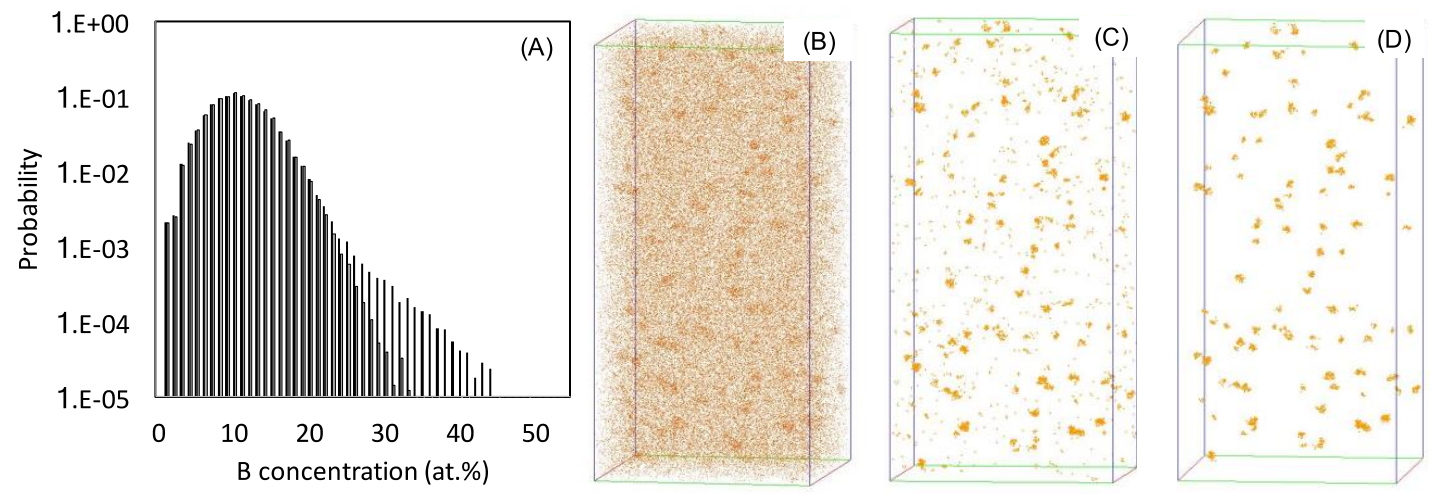

Figure 6: Local concentration measured in $0.75 \mathrm{~nm}$ radius spheres around each $\mathrm{B}$ atom. Open circles are for the random dataset. (A); Overall dataset (B) B atoms with local B concentration > 25 at.\% (C) identified clusters using maximum separation method on the B atoms shown in (C) (D). 


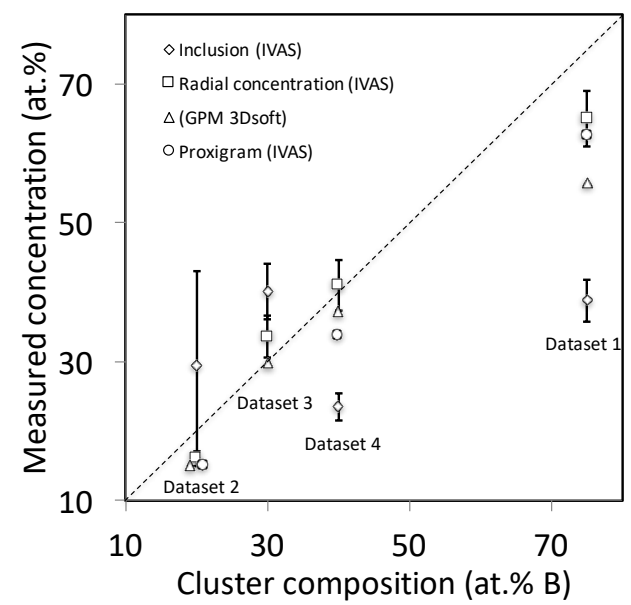

Figure 7: Reported versus nominal compositions for the four datasets.

\section{DISCUSSION \& RECOMMENDATIONS}

The results of this round robin experiment show that a narrower range of outcomes with higher fidelity are reported for microstructures with high solute density contrast, and significantly wider spread are reported for more complex microstructures (cluster proximity or concentrated matrix composition). The synthetic datasets used in this study highlight a number of limitations in the application of the maximum separation method as implemented in the IVAS software, which is still the most widely applied analysis method in the literature. Not all microstructures are amenable to this method, and its application becomes questionable for dataset where the matrix composition is no longer dilute. In this case, other methods may be applied; we illustrate here how the use of local composition to filter solute atoms increases the contrast between matrix and clusters, providing more stable and reliable answers. The selection of the appropriate cluster analysis method is crucial to understanding the microstructure.

We also note that effects of commonly observed artefacts on the quality and stability of the outcomes were not tested in the present experiment but should be taken into account. In particular, the effect of density variations (both in terms of overall density or relative solute density), such as those observed at crystallographic poles or zone lines, should be carefully considered in future analyses. At times, however, relative changes in density due to evaporation artefacts can play in our favor, as in the case of lowerevaporation-field clusters that are reconstructed with increased atomic density, thereby increasing the contrast in comparison to matrix solute atoms. We also did not test whether these methods would be amenable to microstructures with multiple populations of clusters of different solute densities.

\subsection{Cluster detection and counting}

In light of the present findings, we suggest following a workflow to remediate the lack of information reporting and to provide guidance for researchers new to the field of APT. This workflow is relevant to the use of the maximum separation and iso-position methods.

- High contrast datasets may be analyzed by the maximum separation method as implemented in the IVAS software while lower contrast dataset would be best analyzed using concentration filtering, followed by the maximum separation method as implemented in the IVAS software or by the GPM 3Dsoft iso-position method.

- For both methods, because of the strong dependence of the outcome on values of the analysis parameters, a description or justification of the methods by which parameters are selected is required for completeness, so the results can be reproduced. In addition, a sensitivity analysis with respect to the parameters is highly recommended as additional justification for the selection of the parameters' values and as a measure of the uncertainty. 
- In both methods, an approach to selecting values of $\mathrm{d}_{\max }, \mathrm{O}, \mathrm{N}_{\min }$ or $\mathrm{C}_{\mathrm{th}}$, based upon comparison with randomized datasets, to minimize the number of random occurrences, is recommended. We note here that the concentration of the random dataset may be considered carefully when the overall solute concentration is not dilute. The use of a random dataset keeping the overall solute concentration might artificially increase the detection of random clusters.

- Once parameters are set and selected, a check of the randomness of the matrix could be valuable [33] as well as visual inspection.

- In the case of clusters that are close spatially, there is no automated procedure to correct for possible merging of clusters. One may use either manual visual inspection or automated measurements of size, aspect ratio, or concentration to determine the likelihood that the cluster resulted from a merge.

Additional steps, if performed, could provide further information and quantification of uncertainty. These include the use of several instances of a random solid solution to highlight the range of $\mathrm{N}_{\min }$ values that should be considered when reporting the number and size of clusters (Fig. 8).

\subsection{Cluster composition}

Concentration measurements were most susceptible to the selected analysis methods due to the blurred cluster interfaces. Small clusters and precipitates are prone to evaporation artefacts, such as preferential evaporation or retention leading to change in curvature and to non-uniform projection with overlap of matrix and cluster atoms over distances that may be comparable to the average cluster size. In the best-case scenario, this leads to a broadening of the interface while the cluster's core still reflects the original composition. In the vast majority of cases, the reconstructed width of an interface is 2 to $3 \mathrm{~nm}$ even though the structure of interface might be atomically sharp by another imaging technique. It is therefore evident that the accurate quantification of the composition of clusters within this size range may be challenging. Here, evaporation artefacts were enhanced by varying the delocalization of the matrix and cluster atoms. While the maximum separation method is reasonable for determining the number and locations of clusters in high contrast microstructures, it is not recommended for compositional measurements. The iso-position method and the radial concentration profile provided more accurate answers. Here again, however, we emphasize the need for thorough reporting of the method by which composition is measured (e.g. radius over which composition is assessed in the case of the radial concentration profile) and for provision of additional data that will enable future analysis and interpretation. A minimum requirement would be the composition profile averaged for all clusters and the dependence with the maximum core composition with cluster size.

Because the inclusion and erosion methods have been used in a large number of studies, it is worth discussing the effects of the inclusion and erosion parameters if one were to interpret compositional values previously published. The IVAS software limits the value of the erosion parameter, e, to the range of [0, $\mathrm{L}$ ], where $\mathrm{L}$ is the inclusion distance and $\mathrm{L} \leq \mathrm{d}_{\max }$. Given these constraints, for clusters smaller than $2 \mathrm{~nm}$, the nominal cluster composition can rarely be attained for reasonable values of $\mathrm{L} \sim \mathrm{d}_{\max } / 2$, that ensures that most atoms inside the cluster are counted without creating overlap between neighboring clusters. Using Dataset 1 for illustration, all methods systematically underestimated the cluster composition (75\% B; Fig. 7), the reason being the inclusion of matrix atoms along the broad interface. A $\mathrm{d}_{\max }$ value of $0.8 \mathrm{~nm}$ with $\mathrm{N}_{\min }=10$ was selected for the purpose of illustrating the effects of $\mathrm{L}$ and e on the measured cluster compositions. As expected when the value of e increases while keeping L constant (Fig. 9b), the B concentration in the clusters increases due to the removal of the matrix atoms near the interface. The deviation from the target value should scale with the size. With larger clusters, the number of near surface atoms contributing to the deviation gets smaller relative to the number of bulk atoms with the appropriate concentration. In Fig. 9a, where $\mathrm{L}=\mathrm{e}$, one can see that for $\mathrm{L}$ values smaller or equal to $0.2 \mathrm{~nm}$, higher concentrations can be obtained. However, these are artefacts due to the exclusion of A atoms within the clusters, artificially increasing the B concentration. In Dataset 2, different results are observed (Fig. 9c and Fig. 9d). The data were analyzed using a higher order of $\mathrm{O}=5$ with a $\mathrm{d}_{\max }=1.0 \mathrm{~nm}$. For a fixed value of the inclusion distance $\mathrm{L}=1.0 \mathrm{~nm}$, increasing the erosion distance, e, increases the measured average 
concentration but leads to lower concentrations than expected in all cases, except for e $=1.0 \mathrm{~nm}$ at which point the matrix atoms near the surface are removed in excess (Fig. 9c). However, setting $\mathrm{L}=\mathrm{e}$ allows one to find any concentration above the expected concentration (Fig. 9d). The rapid increase in concentration for $\mathrm{L}=\mathrm{e}<0.2 \mathrm{~nm}$ is due to the exclusion of $\mathrm{A}$ atoms that are within the clusters.

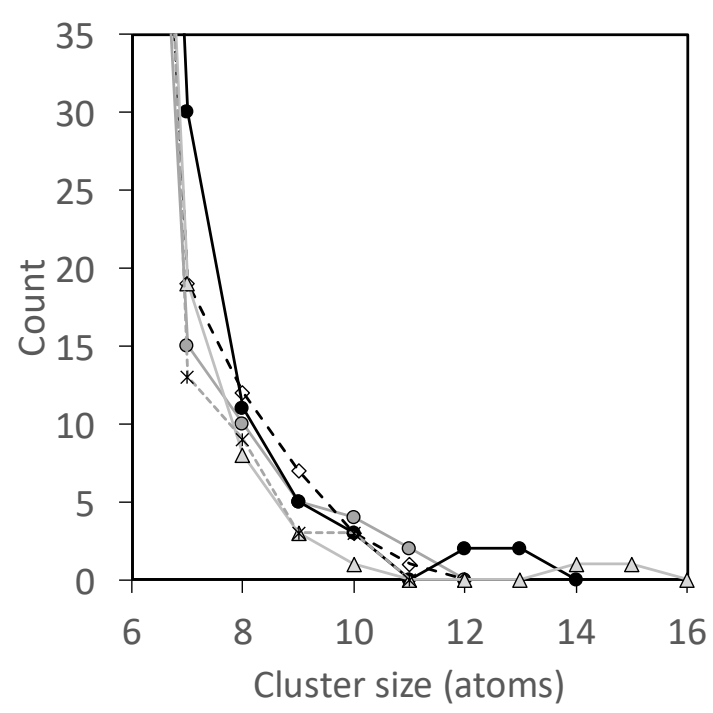

Figure 8: Cluster size distribution for 6 different instances of a random solid solution from Dataset 2 using $\mathrm{O}=5$ and $\mathrm{d}_{\max }=1.0 \mathrm{~nm}$
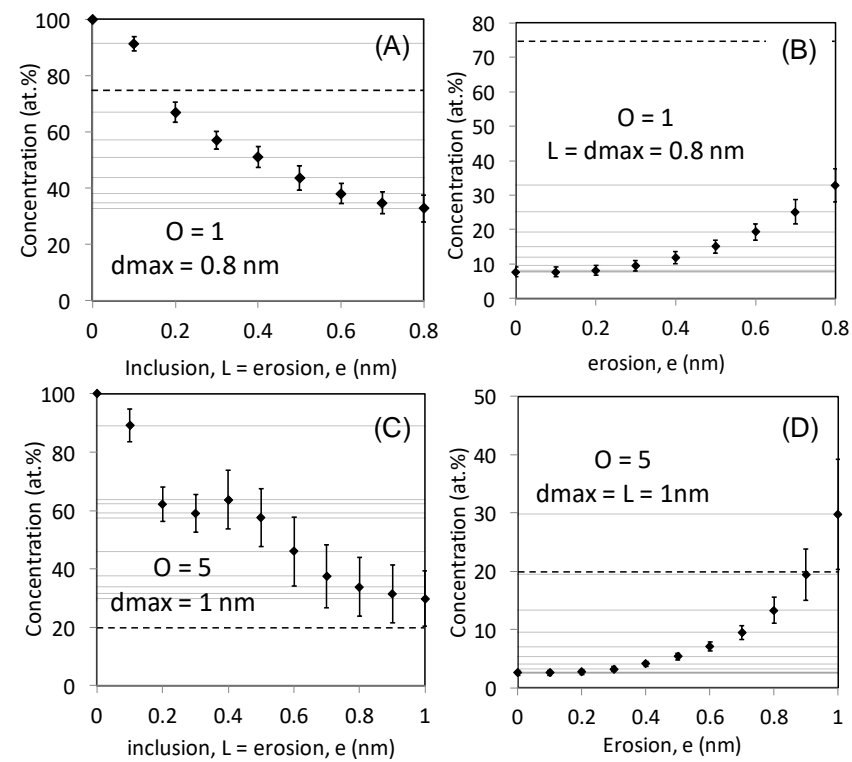

Figure 9: Concentrations measured using the $d_{\max }-\mathrm{L}-\mathrm{e}$ method. Dataset 1 cluster compositions as a function of e when $\mathrm{d}_{\max }=\mathrm{L}=0.8$ $\mathrm{nm}$ (A); Dataset 1 cluster compositions as a function of $\mathrm{e}=\mathrm{L}$ when $\mathrm{d}_{\max }=0.8 \mathrm{~nm}(\mathrm{~B})$. Dataset 2 cluster compositions as a function of $\mathrm{e}$ when $\mathrm{d}_{\max }=\mathrm{L}=1.0 \mathrm{~nm}(\mathrm{C})$; Dataset 2 cluster compositions as a function of $\mathrm{e}=\mathrm{L}$ when $\mathrm{d}_{\max }=1.0 \mathrm{~nm}(\mathrm{D})$

\section{CONCLUSIONS}

Ten international research groups independently analyzed four synthetic datasets created to test the reproducibility, accuracy, and validity of two widely used clustering analysis methods, the maximum separation method and the iso-position method, to identify and measure number density, size, and composition of the cluster populations. This round-robin experiment highlighted the ability of the maximum separation distance to reliably find clusters in microstructures with compositionally highly concentrated clusters in a dilute (low background) matrix. For datasets with higher concentration backgrounds, the iso-position method is recommended, as it reduces the microstructure to one with low background, where the maximum separation method can be applied.

We emphasize the need for thorough reporting of the analysis methods, which includes detailed justification of the method and parameters used. The information is not only essential for the reproducibility of the results, but also ensures that the data presented could be accurately interpreted by future researchers. Minimum information would include:

- Core atoms (or relevant mass to charge ranges)

- Analysis method and any variation on the common approach

- Generation of a randomized dataset for comparison with experimental data to select analysis parameters

- Sensitivity analysis to further refine and justify the choice of parameters, and estimating the uncertainty

- Method used to define $\mathrm{N}_{\min }$

- Specification of how edge clusters are counted and how many are found

- Specification of how merged or split clusters were accounted for 


\section{ACKNOWLEDGEMENTS}

The authors acknowledge the voluntary participation by the members of the APT Round Robin and financial support of EPRI and EDF for the analysis of the aggregate results.

\section{REFERENCES}

[1] J. Hyde, M. Burke, B. Gault, D.W. Saxey, P. Styman, K. Wilford, T. Williams, Ultramicroscopy 111 (2011) 676-682.

[2] A. Etienne, B. Radiguet, P. Pareige, J.P. Massoud, C. Pokor, J. Nucl. Mater. 382 (2008) 64-69.

[3] T. Toyama, Y. Nozawa, W. Van Renterghem, Y. Matsukawa, M. Hatakeyama, Y. Nagai, A. Al Mazouzi, S. Van Dyck, J. Nucl. Mater. 418 (2011) 62-68.

[4] Y. Chen, P.H. Chou, E.A. Marquis, J. Nucl. Mater. 451 (2014) 130-136.

[5] D.J. Larson, P.J. Maziasz, I.S. Kim, K. Miyahara, Scripta Mater. 44 (2001) 359-364.

[6] M.K. Miller, E.A. Kenik, Microsc Microanal.10 (2004) 336-341.

[7] B. Gault, M.P. Moody, J.M. Cairney, S.P. Ringer, Atom Probe Microscopy, Springer, 2012.

[8] B. Gault, F. Danoix, K. Hoummada, D. Mangelinck, H. Leitner, Ultramicroscopy 113 (2012) 182-191.

[9] A.R. Waugh, E.D. Boyes, M.J. Southon, Surf. Sci. 69 (1876) 109-142.

[10] D.J. Rose, J. Applied Physics 27 (1956) 215-220.

[11] F. Vurpillot, A. Bostel, E. Cadel, D. Blavette, Ultramicroscopy 84 (2000) 213-224.

[12] M.K. Miller, M.G. Hetherington, Surf. Sci. 246 (1991) 442-449.

[13] F. Vurpillot, A. Bostel, D. Blavette, Appl. Phys. Letters 76 (2000) 3127-3129.

[14] D.W. Saxey, Ultramicroscopy 111 (2011) 473-479.

[15] A. Heinrich, T.a. Al-Kassab, R. Kirchheim, Mater. Sci. Eng. A 353 (2003) 92-98.

[16] D. Vaumousse, A. Cerezo, P.J. Warren, Ultramicroscopy 95 (2003) 215-221.

[17] A. Cerezo, L. Davin, Surf. Interf. Anal. 39 (2007) 184-188.

[18] L.T. Stephenson, M.P. Moody, P.V. Liddicoat, S.P. Ringer, Microsc Microanal. 13 (2007) 448-463.

[19] D. Blavette, S. Chambreland, Journal de Physique Colloques 47 (1986) C7-503.

[20] B. Radiguet, A. Barbu, P. Pareige, J. Nucl. Mater. 360 (2007) 104-117.

[21] J.M. Hyde, A. Cerezo, T.J. Williams, Ultramicroscopy 109 (2009) 502-509.

[22] E. Meslin, B. Radiguet, M. Loyer-Prost, Acta Mater. 61 (2013) 6246-6254.

[23] F. Vurpillot, F. De Geuser, G. Da Costa, D. Blavette, J. Microscopy-Oxford 216 (2004) 234-240.

[24] F. De Geuser, W. Lefebvre, D. Blavette, Phil. Mag. Letters 86 (2006) 227-234.

[25] L. Couturier, F. De Geuser, A. Deschamps, Mater. Charact. 121 (2016) 61-67.

[26] W. Lefebvre, T. Philippe, F. Vurpillot, Ultramicroscopy 111 (2011) 200-206.

[27] O.C. Hellman, J.A. Vandenbroucke, J. Rüsing, D. Isheim, D.N. Seidman, Microsc Microanal. 6 (2000)

437-444.

[28] M.P. Moody, L.T. Stephenson, A.V. Ceguerra, S.P. Ringer, Microsc. Res. Tech. 71 (2008) 542-550.

[29] J.M. Hyde, E.A. Marquis, K.B. Wilford, T.J. Williams, Ultramicroscopy 111 (2011) 440-447.

[30] P.D. Styman, J.M. Hyde, K. Wilford, G.D.W. Smith, Ultramicroscopy 132 (2013) 258-264.

[31] C.A. Williams, D. Haley, E.A. Marquis, G.D. Smith, M.P. Moody, Ultramicroscopy 132 (2013) 271-278.

[32] R.K. Marceau, L.T. Stephenson, C.R. Hutchinson, S.P. Ringer, Ultramicroscopy 111 (2011) 738-742.

[33] E.A. Jaegle, P.P. Choi, D. Raabe, Microsc. Microanal. 20 (2014) 1662-1671.

[34] R.P. Kolli, D.N. Seidman, Microsc Microanal. 13 (2007) 272-284.

[35] D.J. Larson, T. Prosa, R. Ulfig, B. Geiser, T.F. Kelly, Local Electrode Atom Probe Tomography. Springer, 2014.

[36] W. Lefebvre-Ulrikson, F. Vurpillot, X. Sauvage, Atom Probe Tomography Put Theory into Practice, Elsevier, 2016.

[37] D. Haley, 3Depict - Visualisation \& Analysis for Atom Probe, 2010.

[38] T. Philippe, F. De Geuser, S. Duguay, W. Lefebvre, O. Cojocaru-Miredin, G. Da Costa, D. Blavette, Ultramicroscopy 109 (2009). 